LAW RENCE LIVERMORE N A T IO N A L LABORATORY

The SNM Scanner: A Non-invasive Protocol for Effective Monitoring of Special Nuclear Material Inventories

R. G. Lanier, L. S. Dauffy

May 26, 2004 
This document was prepared as an account of work sponsored by an agency of the United States Government. Neither the United States Government nor the University of California nor any of their employees, makes any warranty, express or implied, or assumes any legal liability or responsibility for the accuracy, completeness, or usefulness of any information, apparatus, product, or process disclosed, or represents that its use would not infringe privately owned rights. Reference herein to any specific commercial product, process, or service by trade name, trademark, manufacturer, or otherwise, does not necessarily constitute or imply its endorsement, recommendation, or favoring by the United States Government or the University of California. The views and opinions of authors expressed herein do not necessarily state or reflect those of the United States Government or the University of California, and shall not be used for advertising or product endorsement purposes.

This work was performed under the auspices of the U.S. Department of Energy by University of California, Lawrence Livermore National Laboratory under Contract W-7405-Eng-48. 


\title{
The SNM Scanner: A Non-invasive Protocol for Effective Monitoring of Special Nuclear Material Inventories
}

\author{
Robert G. Lanier and Lucile S. Dauffy* \\ March 6, 2003 \\ Chemical Biology \& Nuclear Science Division, \\ Lawrence Livermore National Laboratory, Livermore, CA 94550
}

\begin{abstract}
We suggest a system of monitoring special nuclear material inventories which uses simple mathematical techniques to compare the gross features of emitted gamma-ray spectra. In this report we develop the techniques necessary to make such spectral comparisons and describe their application. We also apply these ideas and develop inventory confirmation results using a roomtemperature CdTe detector in a real nuclear-material inventory environment.
\end{abstract}

\section{Introduction}

The verification of special nuclear materials (SNM) continues to be a challenging problem. Prior to storage, materials must undergo some form of characterization to insure that they can be later located and accounted for. Material characterization can involve either a destructive assay or a non-destructive assay. A destructive assay requires that a representative sample of the material is extracted and analyzed while a non -destructive assay often uses the discrete "peak" components of the emitted gammaradiation flux for characterization and quantification.

After a sample undergoes assay, and if no further processing is required, the material is labeled, packaged and finally stored in a suitably secured repository. The destructive and/or non-destructive gamma-ray assay results, along with other physical parameters unique to the material (i.e. weight, storage container identification features, chemical composition, etc.), are then entered into a file and these make up the permanent record of the material during its residence in the repository. A number of such items compose a special nuclear material inventory and this collection must be regularly surveyed to insure that the material remains as it was stored and has not been diverted by accident or for malicious intent.

Typical inventory surveys involve simple protocols such as checking the integrity of tamper-proof seals, re-weighing the object and a rudimentary gamma-ray assay. The latter may be semi-quantitative in the sense that selected spectral regions are checked to insure that a signal exists which is consistent with the SNM catalogued. Specifically,

\footnotetext{
* LLNL Glenn T. Seaborg Institute Postdoctoral Fellow
} 
this means that the spectrum is surveyed for individual peaks and their magnitude quantified. From these data a judgment is then made as to whether the peaks observed are consistent with the registry of the inventory item. Additional checks of the entire spectrum may also be made by a human observer to insure that the current spectrum has features which resemble those from the original gamma-ray assay.

The current technology for SNM high-resolution gamma-ray assays involves germanium detectors that must be cooled to cryogenic temperatures by liquid nitrogen. These devices are generally not portable and carry with them significant infrastructure costs. However, even with the use of such high-resolution systems, inventory verification can be complicated by background effects and because the measurements must be corrected for container thickness to get useful results. If the container thickness is significant, the usefulness of a high-resolution system is degraded because the container wall will absorb the peaks of interest. Inventory checks are also done with low resolution $\mathrm{NaI}$ detector systems that can operate at room temperature and which are portable. In using such systems, a compromise is struck between ease of use and the information obtained. For such systems, the resolution is generally insufficient to resolve peaks of interest and one is reduced to simply recording enhanced count rates in spectral regions that are expected to display the SNM gamma-ray signal.

In the present report, we describe a new SNM inventory verification protocol that uses the emerging technology of CdTe and CdZnTe crystals as gamma-ray detectors. This "Confirmatory Protocol" makes uses of a simple analysis of gamma-ray spectrum taken with these detectors. The combination of the hardware and software described here can likely grow in sophistication but the ideas are simple, easy to understand and, it is hoped, easy to be accepted by the Nuclear Safeguard community.

\section{Information Content of Spectra}

A measured gamma ray spectrum is a discrete distribution that graphs the number of gamma-ray photons detected as a function of energy. That is,

$$
y_{i}=f\left(x_{i}\right)
$$

where $y_{i}$ is the number of photons recorded in channel position $x_{i}$ and where the $x_{i}$ can be instrumentally adjusted to be linear in photon energy:

$$
E=a x_{i}+b \text {. }
$$

The spectrum $y_{i}$ is characterized by a series of very narrow, nearly Gaussian peaks resting on a continuum that resembles a broad but skewed Gaussian distribution. Detailed information about the radiation emitter is contained in the sharp peaks while information about the source environment and its physical composition resides primarily 
in the continuum. The continuum also possesses information about how gamma rays interact with the detector.

Spectrum-stripping algorithms have been developed that find, identify and, when closelying multiplets are encountered, deconvolute the sharp Gaussian peaks. Combined with suitable calibration techniques, a stripping analysis provides accurate assays of radioisotope materials. Ideally, best results are obtained when the radiation detector provides good differentiation between events (i.e. good energy resolution) and when the spectrum has been accumulated for a sufficiently long time. Both factors serve to enhance "signal to noise ratios" and provide peaks with good statistics - i.e. peaks that protrude well above the broad continuum. Factors that inhibit and distort this sort of analyses are thick-walled containers; radioisotopic materials that are packaged as dense, inhomogeneous, metallic forms; measurement systems that provide poor energy resolution; circumstances that require short-duration count times, etc. In these cases, peak structure can be perturbed or "washed out" and the resulting analysis is rendered inaccurate and/or non-reproducible.

While a deconvolution analysis is always the method of choice when circumstances allow and when the most detailed information is required, in many cases, the analysis is overly complicated for the question asked. Such a situation arises in inventory control. In most cases the question is simply: "Does storage container A contain the same material in the same amount today as it did yesterday? As it did last week? As it did last month?" In this report, we show that this question can be answered faithfully by a simple and straight-forward protocol that compares spectra taken from the same container at different times.

\section{Operational Assumptions}

The comparison of two spectra, taken from the same container at different times, can faithfully identify disturbed and undisturbed containers but this procedure is not designed to extract detailed information about inventory items. In other words, a spectrum comparison provides a yes-no or go-nogo decision option. Once a disturbed situation is identified, other procedures and /or protocols would be used to identify whether a theft or diversion has occurred and the magnitude of the quantity involved.

The primary operational assumption of the new protocol is that, all other things being equal, any packaged radioactive sample emits a gamma-radiation signature that combines effects due to the following:

(1) the chemical form of the material

(2) the radioisotopic content of the material

(3) the physical mass of the material

(4) how the material is packaged

(5) the variety of substances used to package the material. 
Given the above, an associated corollary is that the details of the radiation field outside any packaged radioactive sample is absolutely unique because no two such radioactive packages are exactly alike in every way. The final assumption is that technology is available which can accurately and reliably sample a container's gamma-radiation field and that this measured radiation profile can be used to consistently identify one package to the exclusion of all others at any time.

The new protocol described here will neither identify individual peaks nor make specific isotopic identifications. It is assumed that such detailed measurements are done outside the confirmation protocol structure. That is, a sample's "isotopics", along with its other physical or administrative attributes, are independently determined and filed in the primary control file. Subsequent radiation measurements - i.e. "confirmatory measurements" - do not have to be at a high level of specific detail to confirm that the item remains in the inventory undisturbed.

After the material is packaged and after the detailed "isotopics of the sample" have been determined and recorded, an additional measurement of the sample's radiation field is made using a lower resolution portable system. This "fiducial" spectrum represents the totality of the sample's exterior radiation field and represents the initial "fingerprint" of the sample. A second measurement of the sample's radiation field will be made at a later time. The sample is considered undisturbed when the second spectrum is judged identical to the stored "fiducial" spectrum.

The assumptions above represent ideal constructs that must be modified in practice. First, because every radioactive sample possesses and intrinsic half-life, the radiation field will vary with time. Table 1 presents a partial list of controlled nuclear materials and their characteristic half lives.

Table 1: Partial Summary of Controlled Nuclear Materials

\begin{tabular}{|l|l|l|}
\hline $\begin{array}{l}\text { Controlled } \\
\text { Radioisotope }\end{array}$ & Half-life [yrs.] & $\begin{array}{l}\text { Six - month decay } \\
\text { fraction }\end{array}$ \\
\hline & & \\
\hline & & \\
\hline${ }^{235} \mathrm{U}$ & $7.038 \mathrm{E} 08$ & $<1 \mathrm{E}-10$ \\
\hline${ }^{236} \mathrm{~Np}$ & $1.150 \mathrm{E} 05$ & $3.0 \mathrm{E}-06$ \\
\hline${ }^{237} \mathrm{~Np}$ & $2.140 \mathrm{E} 06$ & $1.6 \mathrm{E}-07$ \\
\hline${ }^{238} \mathrm{Pu}$ & $8.774 \mathrm{E} 01$ & $3.9 \mathrm{E}-03$ \\
\hline${ }^{239} \mathrm{Pu}$ & $2.413 \mathrm{E} 04$ & $1.4 \mathrm{E}-05$ \\
\hline${ }^{240} \mathrm{Pu}$ & $6.537 \mathrm{E} 03$ & $5.3 \mathrm{E}-05$ \\
\hline${ }^{241} \mathrm{Pu}$ & $1.516 \mathrm{E} 01$ & $2.3 \mathrm{E}-02$ \\
\hline${ }^{242} \mathrm{Pu}$ & $3.869 \mathrm{E} 05$ & $9.0 \mathrm{E}-07$ \\
\hline & & \\
\hline & &
\end{tabular}


The table shows that if confirmatory measurements of the above materials are performed within a six-month window, the decay characteristics of the material will not substantially alter the measured radiation field. From the above data, the worst case is ${ }^{241} \mathrm{Pu}$ where the short half-life can be expected to contribute $\sim 2.3 \%$ error when comparing spectra from the same source over a 6 month interval. For short half-life samples $(<50-100 \mathrm{yrs}$.), errors introduced because of radioactive decay can be reduced by making confirmatory measurements at shorter intervals.

A second practical consideration is reproducing the source-detector geometry between measurements ${ }^{1}$. There are two aspects to this problem:

(1) When the source in the container has not been disturbed, the detector must be identically positioned relative to the container for subsequent confirmatory measurements.

(2) When the stored material is likely to be disturbed because the container is to be opened or the storage container is handled in such a way as to increase the probability that the original orientation of the sample might be changed, a "before move" and an "after move" measurement must be made.

The first case represents an engineering problem that can be solved by constructing a suitable detector stand that positions the detector at the same location relative to the packaged material. The second case can be solved by administrative controls. If a container is to be moved and if the sample will be disturbed, then an inventory confirmation measurement must be made before the sample is removed from the repository and a new fiducial measurement made after it is returned to storage.

A third problem involves background interferences. At the present time and without more detailed data, it is unclear how background will perturb the confirmatory spectral measurements suggested here. However, initial data suggest that, as long as a container undergoing measurement delivers an overwhelmingly strong radiation signal, the vault background may not be a serious interference and need not be accounted for. A small [2mm x $2 \mathrm{~mm} \times 1 \mathrm{~mm}]$ CdTe detector was used for initial proof-of- principle studies and we found that the vault background had little effect on the results. Our data suggest that, by using a suitably shielded detector, confirmatory measurements may be performed without removing the container from its shelf location in the storage facility.

A more prudent setup for confirmation measurements, however, might involve building a mobile stand that can reside in one corner of a storage vault. When confirmation measurements are requested, sealed cans are removed from their shelf location and reproducibly positioned on the assembly. The mobile stand has a shielded mount to accept the detector probe and to position it reproducibly relative to the container. After

\footnotetext{
${ }^{1}$ In Appendix A, we explore the problem in somewhat more detail.
} 
the measurement operations are complete, the assembly can be wheeled out and stored in another location.

The last problem involves instrument limitations. These require that special care be exercised in selecting and using devices that will be employed for inventory confirmation applications. Since the confirmation protocol will rely on comparison measurements of the integrated radiation profile from a source, a calibration procedure must be developed that will insure that such comparisons are done with confidence. In principle, this is an engineering problem that can be solved with suitably designed hardware. The hardware should be easily "tunable" so that an instrument can be adjusted to deliver a consistent response to a standard radiation source. Finally, applying the hardware in the proper way can be handled with administrative controls and wellwritten procedures. Ideally, instrument adjustments and measurements would be designed so that minimally trained personnel could carry out the confirmation protocols.

The detector and electronics envisioned for the new confirmation measurements combines a small room-temperature detector with a miniature multi-channel analyzer. The unit is intrinsically portable and can be stored in a desk drawer.

\section{Theoretical Discussion}

The comparison of two gamma-ray spectra can be done easily by comparing the amplitudes provided by either a wavelet or a Fourier decomposition procedure. Wavelet decomposition, for example, is used in communications applications where it is necessary to reduce the complex structure in a signal (i.e. voice, video, etc.) to its most basic features. These features are then transmitted to a receiver where a faithful copy of the original is reconstructed. Wavelet decomposition has proven to be a good procedure to reduce information quantity without loss of information content. Therefore, it provides a truncated but faithful basis on which two spectra (signals) can be compared.

While detailed decomposition techniques are faithful bases for spectrum comparisons, it remains to be seen whether they are necessary for the application described in the present report. An alternate way to look at the problem is to extract simple "descriptors" or "comparators" from a spectrum and then use these as a comparison basis. We define a descriptor/comparator ${ }^{2}$ as some number extracted from a spectrum that is meaningfully associated with that spectrum. ${ }^{3}$ To make the definition finer, we define a "unique descriptor" as a quantity associated with one spectrum to the exclusion of all others.

\footnotetext{
${ }^{2}$ The terms "descriptor" and "comparator" are used interchangeably in this report.

${ }^{3}$ As defined here descriptor/comparator is more general than an alternate term that might be associated with, for example, a Taylor's Series expansion coefficient. That is, descriptor/comparator includes the universe of all expansion coefficients as well as other quantities that are not part of this class of real numbers but nevertheless capture some feature of a distribution, i.e. count rate.
} 
We can arbitrarily subdivide descriptors into two classes: "Gross descriptors" and "discrete descriptors". The former captures elements of a spectrum that have an average or "total" quality about them. Examples include the simple average of all the data or some measure of the various symmetries or asymmetries that might exist. Discrete descriptors capture local features in a spectrum. These include sharp features such as individual gamma-ray peaks or Compton-scattering cut-offs. In general, because of statistical fluctuations in the measured data, no descriptor will be unique. That is, within experimental error, if two spectra have the same single descriptor, they are not necessarily obtained from the same sample. However, as we increase the number of different descriptors, it will be progressively unlikely that two different spectra will exhibit the same set of descriptors. In this document, we suggest that a set of six descriptors can be identified that allow for a credible basis for comparing spectra. In the discussion that follows, we present these ideas in more rigorous detail.

We start by noting that the distribution described in Eqn. (1) can be normalized to time ${ }^{4}$ and transformed into a probability distribution. Thus, take

$$
\&=\frac{y_{i}}{t}=\&\left(x_{i}\right)
$$

and

$$
A=\square_{i}^{n} \frac{y_{i}}{t}=\prod_{i}^{n} \&
$$

and form

$$
Y\left(x_{i}\right)=\& / A=\&\left(x_{i}\right) / A
$$

where $Y\left(x_{i}\right)$ now has the properties of a probability density distribution with $x_{i}$ as the random variable. That is

$$
\begin{aligned}
& \text { (a) } Y\left(x_{i}\right) \geq 0 \\
& \text { (b) } \square Y\left(x_{i}\right)=1 \text { with } \square \quad \square x_{i} \square \\
& \text { (c) } P\left(x_{i}\right)=Y\left(x_{i}\right) \text { where } 0 \quad \square \quad P\left(x_{i}\right) \quad \square 1
\end{aligned}
$$

\footnotetext{
${ }^{4}$ The time parameter is almost redundant because we are comparing ratios of ratios and the time will usually cancel out. We include the time parameter to avoid confusion and to emphasize that comparing counts per unit time on a channel by channel basis is the proper way to compare gamma ray spectra given the experimental conditions assumed in this report. However, this is not true when count rates or measures of count rate are compared.
} 
so that $P\left(x_{i}\right)$ is the probability that an event is registered in $x_{i}$ per unit time. The moment-generating function of a discrete random variable $x_{i}$ is given by ${ }^{5}$

$$
m(\square)=\square_{i=1}^{n} e^{x_{i} \square} Y\left(x_{i}\right)=1+\square \square+\square_{2} \frac{\square^{2}}{2 !}+\square_{3} \frac{\square^{3}}{3 !}+\mathrm{L}
$$

where $\square_{i}$ are the moments of the probability distribution described by Eqn. (4) and $\square$ is an arbitrary parameter. The individual moments are given by

$$
\square_{m}=\square_{i=1}^{m} x_{i}^{m} Y\left(x_{i}\right)
$$

Consider a second probability density distribution (spectrum) $\mathrm{Z}\left(x_{i}\right)$ normalized to time $t \square$, where in general, $t \neq t \square$ then

if and only if

$$
\mathrm{Y}\left(x_{i}\right)=\mathrm{Z}\left(x_{i}\right)
$$

$$
\square_{m}=\square_{m}
$$

for all $m$, where $\square_{m}$, $\square_{m}$ are the moments of $\mathrm{Y}\left(x_{i}\right)$ and $\mathrm{Z}\left(x_{i}\right)$, respectively. That is to say that we can compare two distributions through their moments. Or, said another way, the moments extracted from two spectra are gross descriptors and when compared, provide some measure of the similarity or dissimilarity of two spectra.

Identifying a set of discrete descriptors from a spectrum is less straight-forward than extracting the moments of a spectral distribution. Excluding low order wavelet or Fourier decomposition amplitudes, a simpler set of discrete descriptors might include the ratio of the spectrum area to its perimeter, the spectrum's fractal dimension, etc. For the present discussion, we evaluate the following simple discrete descriptor which is based on looking at a spectrum in a somewhat different way. We also obtain an additional gross descriptor from the analysis.

Without any loss of information, we rewrite Eqn. (2) as a multidimensional vector thus,

$$
\begin{aligned}
\& & =\& \square_{1}+\& \square_{2}+\mathrm{L} \& \square_{i} \\
& =\&\left(x_{1}\right) \square_{1}+\&\left(x_{2}\right) \square_{2}+\mathrm{L} \&\left(x_{i}\right) \square_{i}
\end{aligned}
$$

\footnotetext{
${ }^{5}$ See, for example, Handbook of Tables for Probability and Statistics, $2^{\text {nd }}$ edition, William H. Beyer, editor, CRC Press, Inc, Boca Raton, FL, 1985. pp 15 - 18.
} 
where $\square_{i}$ are unit vectors over a space of dimension $n$. In other words, we represent the time normalized spectrum as an ordered $n$-tuple of real numbers. The magnitude of $\&$ is given by

$$
\| r_{d}^{r}=\sqrt{(\& \&)}=\sqrt{\prod_{i=1}^{n} \& \&^{2}}
$$

from which we can compute

$$
\hat{k}_{y}^{r}=\prod_{i=1}^{n}
$$

where $\hat{k}_{y}^{r}$ is a unit vector collinear with $\&$. Consider a second vector (spectrum)

$$
\stackrel{r}{\&}=\prod_{i=1}^{n} \& \square_{i} \equiv \prod_{i=1}^{n} \&\left(x_{i}\right) \square_{i} \text {, }
$$

with it's associated unit vector

$$
\hat{j}_{z}^{r}=\bigsqcup_{i=1}^{n} \frac{1}{r}
$$

where

$$
\&=z_{i} / t \square
$$

and where, in general, $t \neq t$. A necessary and sufficient condition that

$$
\text { '\& = '\& }
$$

(i.e. that the spectra are identical) requires that the following be true:

$$
\hat{k}_{y}^{r} \cdot \hat{j}_{z}^{r}=1 \text { and } \quad\|\stackrel{1}{\prime}=\|^{\prime} \quad(10) \text {. }
$$

In other words, the unit vectors derived from the two spectral vectors must be collinear (i.e. their inner product $=1$ ) and, the overall magnitude of the vectors must be equal.

Eqns. (6) and Eqns. (10) are a set of $m+2$ comparators that can be used to compare spectra. As a working hypothesis, we take six comparators from this set and claim that 
they provide a sufficient basis on which to compare spectra. For example, the first three moments of the distribution represented by Eqn. (4), are the distribution's centroid, a measure of its width, and the measure of its asymmetry. ${ }^{6}$ Eqns. (10) are a direct measure of the channel-by-channel similarities as well as a relative measure of total count rate. ${ }^{7}$

Once the six descriptors are calculated for two spectra, we compare them as ratios and determine their proximity to unity. That is, in an ideal case, the individual ratios of the descriptors will be unity and any measure of their proximity to unity will be zero. More precisely, let

$$
R_{i}=d_{i} / d \square
$$

where $d$ is an arbitrary descriptor calculated from two spectra measured from the same item but where one spectrum was accumulated for time $t$ and the other for time $t$. Ideally, $R_{i}=1$ but, because of measurement error, in general, $R_{i} \neq 1$ but ought to be close to it. Thus, we expect that

$$
\square_{i}=\left|1 \square R_{i}\right|=\left|1 \square^{d_{i}} / d \square\right| \square 0
$$

is a measure of that proximity. For the entire set of six descriptors, we calculate

$$
\square=\sqrt{\frac{\prod_{i}^{5} \square_{i}^{2}}{6}}
$$

Eqn. (11) represents the standard deviation of how well the descriptors line up with unity. Ideally, $\square=0$ but, for the present study, we arbitrarily set the level at $\square \square 0.1$. From the comparisons, performed here, this turns out to be a very stringent criterion.

Given reasonable measurement statistics, the probability that the six quantities defined by Eqns. (6) and Eqns. (8) will be the same for two different samples is sufficiently small so that items can be reliably distinguished. However, even if the protocol cannot confidently distinguish between two different containers, such a chance occurrence will also be difficult to separate by other techniques based on gamma-ray spectrum analysis techniques. Nevertheless, this should not invalidate the protocol because alternate physical or administrative means may be used to make a differentiation. In summary,

\footnotetext{
${ }^{6}$ We emphasize that all moments for the present application are taken with respect to the spectrum origin. If, for example, the $2^{\text {nd }}$ moment is taken with respect to the centroid, it is referred to as the "variance" of the distribution.

${ }^{7}$ The total count rate is, of course, given by Eqn. (3).
} 
for the majority of the inventory items, we claim that an automated procedure based on the ideas of gross and discrete descriptors can vastly reduce the human interaction with confirmatory measurements.

\section{Measurement Hardware}

The prototype system used an AMP-TEK Corporation "ROVER" unit (Figure 1) with an electrically cooled CdTe detector crystal $(2 \mathrm{~mm} \times 2 \mathrm{~mm} \times 1 \mathrm{~mm})$ to measure the radiation fields around a set of selected source materials. Because the crystals are small, they are significantly less sensitive relative to liquid-nitrogen-cooled Ge systems or to room-temperature $\mathrm{NaI}$ systems. Nevertheless, for the application described here, low sensitivity may be an advantage.

The ROVER is a self-contained, portable multi-channel analyzer radiation-detection system. It contains electronics for cooling the CdTe crystal and is fitted with a HewletPackard 200LX pocket computer for data accumulation and low-level instrument control, data analysis and data storage. The unit weighs $\sim 2 \mathrm{lbs}$ and is hand held. In the present studies, the data were taken and stored on the ROVER unit and analyzed off line on a lap-top personal computer (PC). A special computer program was written to sort through the data and to make the spectrum comparisons outlined in the previous section - i.e. the "confirmatory measurements". The code is written in Microsoft FORTRAN.

In principle, the process could be modified so that all operations are performed on the ROVER-attached pocket PC. For the present, it seemed more appropriate to accumulate the data in the ROVER unit and transfer it for later analysis to a PC. In a real environment where inventory confirmations are being carried out, it would likely be the case that a two-computer protocol would be more sensible and would better satisfy security concerns. That is, data would be first accumulated with the ROVER unit by a storage-vault worker. Then, after data accumulation, a second operator would accept the flash-memory card from the accumulation system and transport it to another computer. The second computer would be connected to the inventory data base and have direct read-only access to it. The confirmatory checks would then be run independent of the accumulation function. A

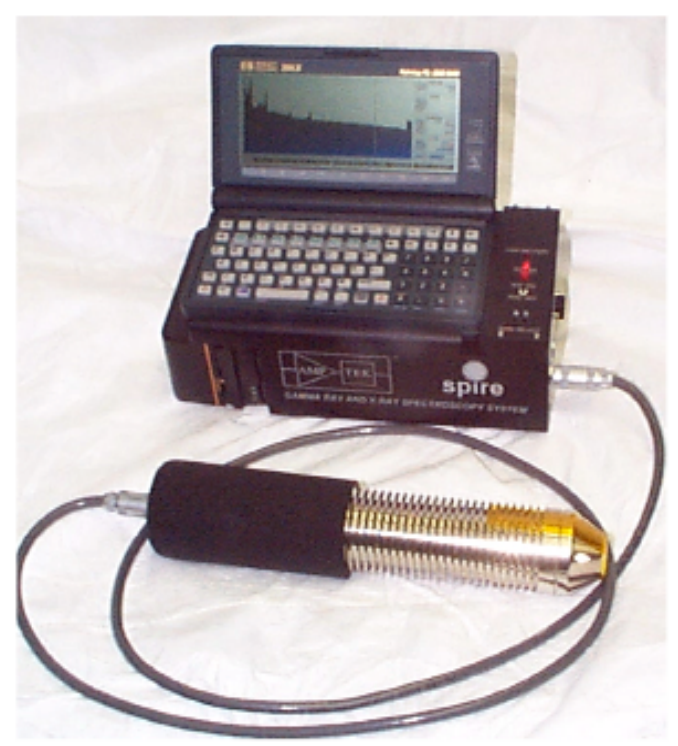

Figure 1: The Rover Unit used in the confirmation measurements

\footnotetext{
${ }^{8}$ AMPTEK Corporation, 6 De Angelo Drive, Bedford, MA 07130 USA
} 
suggested operational flow chart, which organizes the process control, is offered in Figure 2.

\section{Description of the Prototype Measurements}

The ROVER device was used to exercise a "Confirmation Test Protocol" to test the confirmation technique. Table II summarizes important details of the materials that were selected for this study.

Table II lists a random selection of miscellaneous radioisotope calibration materials as well as a series of samples that were controlled inventory materials. The calibration materials were radioactive sources that are easily handled and easy to use in a radiationmeasurement laboratory environment, i.e. the sources were simply packaged and could be identified as "hand-held" and/or "hand-portable". These included a lantern mantel which contains naturally radioactive ${ }^{232} \mathrm{Th}\left(1.4 \times 10^{10} \mathrm{yr}\right)$ sealed in a plastic wrapper, an

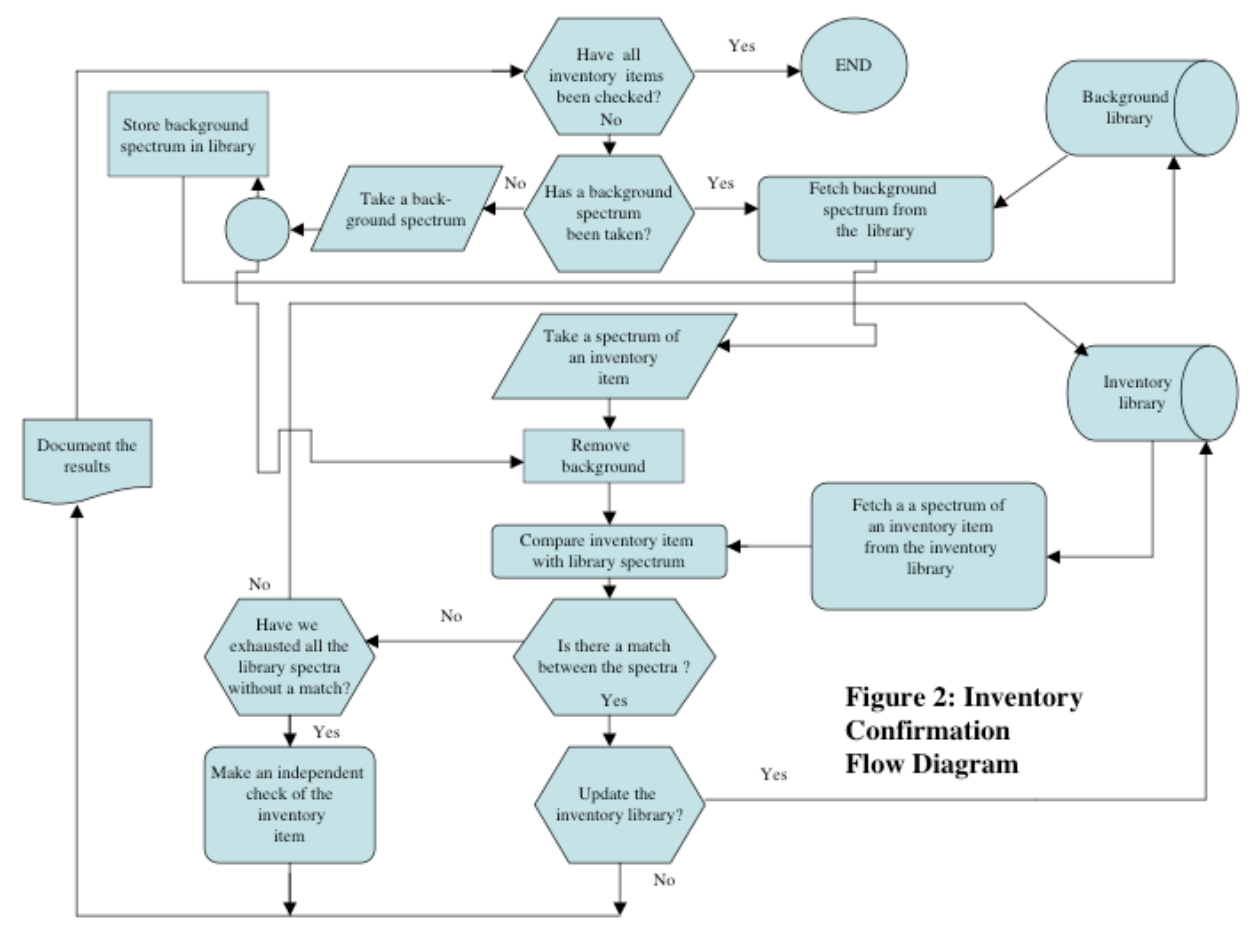

evaporated drop of ${ }^{152} \mathrm{Eu}(13 \mathrm{yr})$ solution sealed between two plastic sheets and mounted on an $3 \mathrm{~cm}$ diameter aluminum ring, a series of four ${ }^{235} \mathrm{U}\left(7.0 \times 10^{8} \mathrm{y}\right)$ oxide standard sources sealed in $\sim 3$ " x 3" diameter stainless steel cans, a standard ("PIDI") ${ }^{239} \mathrm{Pu}(2.4 \mathrm{x}$ $\left.10^{4} \mathrm{y}\right)$ source encapsulated in a $\sim 1$ " $\mathrm{x} 1$ " stainless-steel plug and a small $\sim 1 \mathrm{~cm} \times 1 \mathrm{~cm}$ rectangular plastic-encapsulated source of ${ }^{57} \mathrm{Co}(270 \mathrm{~d})$. The measurement of these 
sources was performed under controlled conditions and in a low background environment. The detector was fixed on a stationary laboratory bench and a ring stand held the radioactive source. The source could then be moved relative to the detector and positioned in a controlled way.

The special nuclear material inventory items were measured during a one-hour limited access visit to a controlled nuclear-material vault. The sources were a series of nine metal cans (identified in Table II, Column 3 by a "Vault" prefix) that are designed for special nuclear-material storage. The selection of cans was purely random but there was an attempt to insure that the selection provided a realistic profile of the materials stored in the vault.

From both sets of measurements, a fictitious sample library was constructed which provided the data base against which subsequent new spectra could be checked. Table II, Column 3, is identified as that data base.

In the process of compiling the library, a number of samples were measured a second

\begin{tabular}{|c|c|c|c|c|}
\hline & & & & \\
\hline & & & & \\
\hline & & & & \\
\hline & Table II: & & Inventory Control tests & \\
\hline $\begin{array}{l}\text { Description } \\
\text { General }\end{array}$ & Date & Spectrum ID & Comments & $\begin{array}{c}\text { Count Time } \\
{[\mathrm{sec}]}\end{array}$ \\
\hline & & & & \\
\hline Laboratory & $11 / 8 / 02$ & C071108B & Co 57 Source & 620.80 \\
\hline Test & & C0601108 & Co 57 Source & 305.00 \\
\hline Samples & & U23519A & $1.9 \% 235 \mathrm{U}$ source & 7431.80 \\
\hline & & U23545A & $4.5 \% 235 \mathrm{U}$ source & 4981.50 \\
\hline Controlled & & U23545B & $4.5 \% 235 \mathrm{U}$ source & 7554.70 \\
\hline Environment & & U23587A & $2.9 \% 235 \mathrm{U}$ source & 81030.80 \\
\hline & & MAN1107A & Lantern Mantel & 70618.00 \\
\hline Misc & & MANT0918 & Lantern Mantel & 96891.00 \\
\hline Calibration & & MANT0919 & Lantern Mantel & 84801.00 \\
\hline Materials & & MANT0920 & Lantern Mantel & 141176.00 \\
\hline & & EUCAL & Eu Calibration Source & 173818.00 \\
\hline & $11 / 11 / 02$ & MAN1111A & Lantern Mantel & 246332.11 \\
\hline & & U235295B & $2.9 \% 235 \mathrm{U}$ source & 10675.00 \\
\hline & & U23529C & $2.9 \% 235 \mathrm{U}$ source with stainless absorber & 65050.90 \\
\hline & & U23529D & $2.9 \% 235 \mathrm{U}$ w/o stainless absorber & 12683.55 \\
\hline & & PUS1112A & PIDI Standard short count/1.5" thick paraffin & 649.31 \\
\hline & $11 / 12 / 02$ & PUS1112B & PIDI standard longer count/1.5" thick paraffin & 6613.81 \\
\hline & $11 / 13 / 02$ & PUS1113A & PIDI Standard/ paraffin is $17 / 8 "$ thick & 58725.55 \\
\hline & $11 / 13 / 02$ & PUS1113B & $\begin{array}{c}\text { PIDI Standard/ same as above/but paraffin is } 1.5 \\
\text { " thick }\end{array}$ & 9808.29 \\
\hline Inventory & $11 / 13 / 02$ & Vault01 & unmarked vault item & 320.45 \\
\hline Items & & Vault02 & $"$ & 134.95 \\
\hline & & Vault03 & " & 243.21 \\
\hline Storage & & Vault04 & $"$ & 166.95 \\
\hline Vault & & Vault05 & $"$ & 288.6 \\
\hline Environment & & Vault06 & $"$ & 60.33 \\
\hline & & Vault07 & " & 235.71 \\
\hline Controlled & & Vault08 & $"$ & 218.13 \\
\hline Special & & Vault09 & " & 129.01 \\
\hline Nuclear & & Vault10 & $"$ & 150.93 \\
\hline Materials & & Vault11 & $"$ & 142.29 \\
\hline & & & & \\
\hline & & & & \\
\hline & & & & \\
\hline
\end{tabular}


time. These "repeat" measurements were used to query the library to mimic how a confirmation measurement would be done. Under these conditions, it was always possible for the computer program to identify a source in the library. Once an initial spectrum from a source was accumulated and placed into the library, it was always possible to make a second measurement and unambiguously identify that source as being in the library.

\section{Measurement Results}

Table III A, B, C and Table IV summarize some of the results of the "confirmation measurements". Figures $3 \mathrm{~A}, \mathrm{~B}$ and $\mathrm{C}$ and Figure 4 display the spectra compared in Tables III - IV. The measurement conditions (i.e. source-detector distance, position in the vault, etc.) associated with each spectrum in the library was identified and recorded

\begin{tabular}{|c|c|c|c|c|c|c|c|}
\hline Table IIIA & & & & & & & \\
\hline & & & & & & & Match exists \\
\hline & & & & & & & if Final \\
\hline \multicolumn{2}{|l|}{ Queried Spectrum } & \multicolumn{2}{|c|}{ Mant0920.mca } & \multicolumn{2}{|c|}{$141176.00 \mathrm{Sec}$} & & Comparator \\
\hline & & & & & & & $<\sim 0.10$ \\
\hline $\begin{array}{l}\text { Data Base } \\
\text { Spectrum }\end{array}$ & $\begin{array}{c}\text { First } \\
\text { Moment }\end{array}$ & $\begin{array}{l}\text { Second } \\
\text { Moment }\end{array}$ & $\begin{array}{c}\text { Third } \\
\text { Moment }\end{array}$ & $\begin{array}{c}\text { Fourth } \\
\text { Moment }\end{array}$ & $\begin{array}{c}\text { Inner } \\
\text { Product }\end{array}$ & $\begin{array}{l}\text { Count } \\
\text { Rate }\end{array}$ & $\begin{array}{c}\text { Final } \\
\text { Comparator }\end{array}$ \\
\hline pus1112a.mca & 2.22 & 5.45 & 13.41 & 17.16 & 20.00 & 20.00 & 13.89 \\
\hline mant0918.mca & 1.43 & 4.26 & 15.19 & 20.00 & 1.34 & 1.27 & 9.78 \\
\hline mant0919.mca & 1.00 & 1.00 & 1.00 & 1.01 & 1.00 & 1.00 & 0.00 \\
\hline eucal1.mca & 1.18 & 2.10 & 5.31 & 15.34 & 1.73 & 14.94 & 8.37 \\
\hline co71108b.asc & 1.20 & 1.58 & 2.48 & 4.49 & 2.60 & 20.00 & 7.94 \\
\hline co601108.asc & 1.19 & 1.57 & 2.42 & 3.86 & 2.63 & 20.00 & 7.90 \\
\hline u23519a.asc & 1.17 & 1.08 & 1.50 & 3.40 & 1.62 & 20.00 & 7.83 \\
\hline u23545a.asc & 1.17 & 1.14 & 1.25 & 2.60 & 1.63 & 20.00 & 7.79 \\
\hline u23545b.asc & 1.17 & 1.14 & 1.25 & 2.62 & 1.64 & 20.00 & 7.79 \\
\hline u23587a.asc & 1.17 & 1.12 & 1.36 & 2.99 & 1.63 & 20.00 & 7.81 \\
\hline man1107a.asc & 1.01 & 1.99 & 6.03 & 20.00 & 1.18 & 3.07 & 8.08 \\
\hline man1111a.asc & 1.03 & 1.84 & 5.46 & 17.98 & 1.21 & 2.96 & 7.22 \\
\hline u235295b.asc & 1.18 & 1.13 & 1.34 & 2.93 & 1.63 & 20.00 & 7.80 \\
\hline u23529C.asc & 1.13 & 1.04 & 1.47 & 3.18 & 1.58 & 20.00 & 7.81 \\
\hline u23529D.asc & 1.18 & 1.12 & 1.36 & 2.98 & 1.63 & 20.00 & 7.81 \\
\hline pus1112b.mxx & 2.22 & 5.49 & 14.36 & 20.00 & 20.00 & 20.00 & 14.62 \\
\hline pu1113ax.mca & 2.23 & 5.53 & 14.40 & 20.00 & 20.00 & 20.00 & 14.63 \\
\hline pu1113bx.mca & 2.24 & 5.55 & 14.53 & 20.00 & 20.00 & 20.00 & 14.65 \\
\hline vault01x.mca & 1.01 & 1.18 & 1.63 & 2.77 & 1.50 & 20.00 & 7.80 \\
\hline vault02x.mca & 2.00 & 3.76 & 5.13 & 3.80 & 20.00 & 20.00 & 11.22 \\
\hline vault03x.mca & 1.13 & 1.12 & 1.04 & 1.50 & 1.44 & 20.00 & 7.76 \\
\hline vault04x.mca & 1.58 & 1.96 & 1.82 & 1.28 & 5.90 & 20.00 & 8.03 \\
\hline vault05x.mca & 1.40 & 1.56 & 1.27 & 1.45 & 2.85 & 20.00 & 7.80 \\
\hline vault06x.mca & 2.09 & 4.70 & 9.82 & 11.98 & 20.00 & 20.00 & 12.49 \\
\hline vault07x.mca & 1.13 & 1.23 & 2.73 & 7.82 & 2.33 & 20.00 & 8.29 \\
\hline vault08x.mca & 1.33 & 1.39 & 1.08 & 1.72 & 2.65 & 20.00 & 7.79 \\
\hline vault09x.mca & 1.36 & 1.42 & 1.11 & 1.58 & 2.44 & 20.00 & 7.79 \\
\hline vault 10x.mca & 1.01 & 1.17 & 1.56 & 2.40 & 1.53 & 20.00 & 7.78 \\
\hline vault 11 x.mca & 2.04 & 4.26 & 7.46 & 7.78 & 20.00 & 20.00 & 11.70 \\
\hline
\end{tabular}

so that when the sample was remeasured, the experimental conditions could be repeated.

In table IIIA, a lantern mantle was measured under a repeatable condition. That is, the source-detector separation was reproduced but the counting time was arbitrary. The spectrum was then compared with the library list and the "final comparator" calculated. Figure IIIA shows the two spectra as appearing nearly identical. The Table IIIA clearly shows that the source is unambiguously identified, i.e. the final comparator is very small. 
Table IIIB and Figure 3B show a similar comparison for two enriched uranium samples.

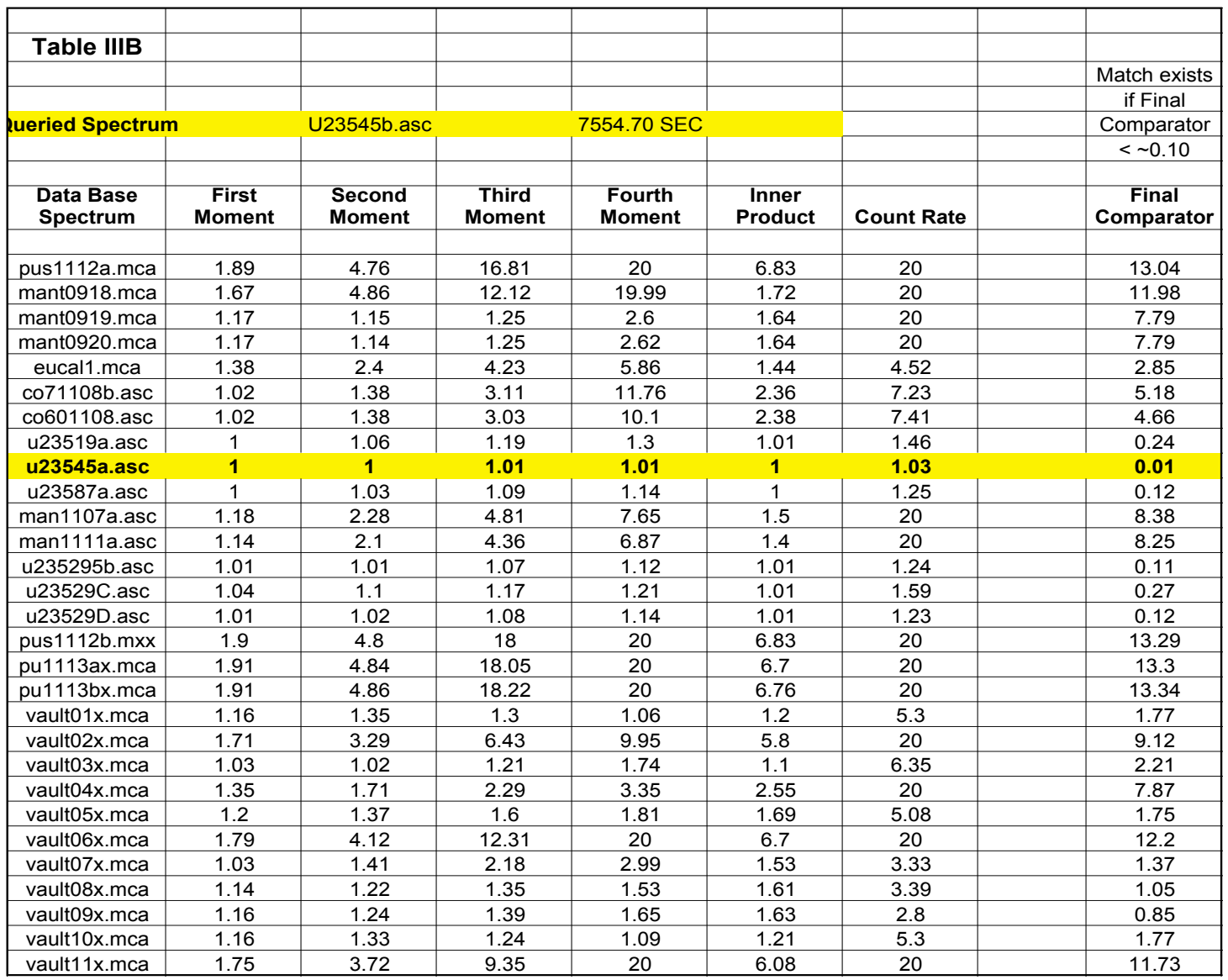

The small comparator shown in Table IIIB indicates clearly that the code easily selected the proper uranium sample from the library. The sample selected, U23545A, is enriched in $235 \mathrm{U}$ to $\sim 4.5 \%$. Other similarly packaged sources in the library are enriched to $2.9 \%$, and $1.9 \%$, and these - quite appropriately - were not selected as a match. Figure 3B also shows the spectrum for the uranium sample enriched in $235 \mathrm{U}$ to $2.9 \%$. Although this spectrum (U23529C) is very similar to the spectrum from the sample enriched to $4.5 \%$ (U23545A and U23545B), its final comparator (0.27) did not fall in the range where it would be considered a match.

The measurements in the storage vault were limited in time, so the spectrum accumulation times were necessarily short, i.e. generally $<5 \mathrm{~min}$. Storage cans were identified at random, removed from the storage shelf and, in most cases, simply measured with the can on the floor directly below the actual storage-shelf location. Table IIIC shows that a duplicate measurement on the sample labeled Vault01X was correctly identified. For comparison in Figure 3C, the spectra Vault04X and its associated comparator (2.36) is also displayed and shows that this item can be easily discriminated

from the material represented by the spectra labeled Vault01X and Vault10X. 


\begin{tabular}{|c|c|c|c|c|c|c|c|}
\hline Table IIIC & & & & & & & \\
\hline & & & & & & & Match exists \\
\hline & & & & & & & if Final \\
\hline \multicolumn{2}{|l|}{ Queried Spectrum } & \multicolumn{2}{|c|}{ Vault 10x.mca } & \multirow{2}{*}{\multicolumn{2}{|c|}{ 150.93 SEC }} & & Comparator \\
\hline & & & & & & & $<\sim 0.10$ \\
\hline $\begin{array}{l}\text { Data Base } \\
\text { Spectrum }\end{array}$ & $\begin{array}{c}\text { First } \\
\text { Moment }\end{array}$ & $\begin{array}{l}\text { Second } \\
\text { Moment }\end{array}$ & $\begin{array}{c}\text { Third } \\
\text { Moment }\end{array}$ & $\begin{array}{c}\text { Fourth } \\
\text { Moment }\end{array}$ & $\begin{array}{c}\text { Inner } \\
\text { Product }\end{array}$ & $\begin{array}{c}\text { Count } \\
\text { Rate }\end{array}$ & $\begin{array}{c}\text { Final } \\
\text { Comparator }\end{array}$ \\
\hline pus1112a.mca & 2.19 & 6.34 & 20.00 & 20.00 & 4.47 & 7.92 & 11.63 \\
\hline mant0918.mca & 1.45 & 3.65 & 9.77 & 20.00 & 1.49 & 20.00 & 11.59 \\
\hline mant0919.mca & 1.01 & 1.16 & 1.55 & 2.39 & 1.53 & 20.00 & 7.78 \\
\hline mant0920.mca & 1.01 & 1.17 & 1.56 & 2.40 & 1.53 & 20.00 & 7.78 \\
\hline eucal1.mca & 1.19 & 1.80 & 3.41 & 6.38 & 1.21 & 20.00 & 8.13 \\
\hline co71108b.asc & 1.18 & 1.84 & 3.86 & 10.79 & 1.85 & 1.36 & 4.20 \\
\hline co601108.asc & 1.18 & 1.83 & 3.76 & 9.27 & 1.88 & 1.40 & 3.60 \\
\hline u23519a.asc & 1.16 & 1.25 & 1.04 & 1.41 & 1.20 & 7.75 & 2.77 \\
\hline u23545a.asc & 1.16 & 1.33 & 1.25 & 1.08 & 1.21 & 5.46 & 1.83 \\
\hline u23545b.asc & 1.16 & 1.33 & 1.24 & 1.09 & 1.21 & 5.30 & 1.77 \\
\hline u23587a.asc & 1.16 & 1.30 & 1.14 & 1.25 & 1.21 & 6.63 & 2.31 \\
\hline man1107a.asc & 1.02 & 1.71 & 3.88 & 8.34 & 1.33 & 20.00 & 8.40 \\
\hline man1111a.asc & 1.02 & 1.58 & 3.51 & 7.48 & 1.24 & 20.00 & 8.26 \\
\hline u235295b.asc & 1.17 & 1.31 & 1.16 & 1.22 & 1.20 & 6.57 & 2.28 \\
\hline u23529C.asc & 1.11 & 1.21 & 1.06 & 1.32 & 1.19 & 8.44 & 3.04 \\
\hline u23529D.asc & 1.17 & 1.31 & 1.14 & 1.24 & 1.20 & 6.54 & 2.27 \\
\hline pus1112b.mxx & 2.20 & 6.39 & 20.00 & 20.00 & 4.40 & 7.89 & 11.63 \\
\hline pu1113ax.mca & 2.21 & 6.44 & 20.00 & 20.00 & 4.15 & 5.27 & 11.41 \\
\hline pu1113bx.mca & 2.21 & 6.47 & 20.00 & 20.00 & 4.14 & 7.54 & 11.59 \\
\hline vault01x.mca & 1.00 & 1.01 & 1.05 & 1.15 & 1.03 & 1.00 & 0.07 \\
\hline vault02x.mca & 1.98 & 4.38 & 7.98 & 9.13 & 4.01 & 20.00 & 9.10 \\
\hline vault03x.mca & 1.12 & 1.31 & 1.50 & 1.60 & 1.08 & 1.20 & 0.36 \\
\hline vault04x.mca & 1.56 & 2.28 & 2.83 & 3.07 & 1.97 & 5.79 & 2.36 \\
\hline vault05x.mca & 1.38 & 1.82 & 1.98 & 1.66 & 1.41 & 1.04 & 0.63 \\
\hline vault06x.mca & 2.07 & 5.48 & 15.27 & 20.00 & 4.39 & 20.00 & 12.64 \\
\hline vault07x.mca & 1.12 & 1.06 & 1.76 & 3.25 & 1.25 & 1.59 & 1.01 \\
\hline vault08x.mca & 1.32 & 1.62 & 1.68 & 1.40 & 1.35 & 1.56 & 0.51 \\
\hline vault09x.mca & 1.35 & 1.65 & 1.72 & 1.52 & 1.35 & 1.89 & 0.61 \\
\hline vault 11 x.mca & 2.02 & 4.96 & 11.59 & 18.70 & 4.06 & 20.00 & 11.64 \\
\hline
\end{tabular}

Table IV and Figure 4 represents a close match between two vault items that are not identical. Based on the calculated comparator $(0.28)$, the code correctly rejects the sample as a true match. But under real conditions, this situation might warrant an additional administrative and/or physical check. because the spectra appear virtually identical in almost every detail. The point of such a comparison is that in this situation, it is likely that even if an analysis of the spectrum is based on a peak stripping algorithm, it would show that, within experimental error, the isotopic content of both cans is nearly the same. Here again, administrative and/or physical controls would be expected to yield additional comparators which would allow proper differentiation of the samples.

Finally, Figure 5 shows a random comparison of spectra from some of the other items in the vault. This comparison shows that there is a wide range of comparators in the inventory items.

\section{Summary}

A system of monitoring special nuclear material inventories is suggested which uses simple mathematical techniques to compare the features of emitted gamma-ray spectra. 


\begin{tabular}{|c|c|c|c|c|c|c|c|}
\hline Table IV & & & & & & & \\
\hline & & & & & & & Match exists \\
\hline & & & & & & & if Final \\
\hline \multicolumn{2}{|l|}{ Rueried Spectrum } & Vault11x.mca & & 142.29 SEC & & & Comparator \\
\hline & & & & & & & $<\sim 0.10$ \\
\hline $\begin{array}{l}\text { Data Base } \\
\text { Spectrum }\end{array}$ & $\begin{array}{c}\text { First } \\
\text { Moment }\end{array}$ & $\begin{array}{l}\text { Second } \\
\text { Moment }\end{array}$ & $\begin{array}{c}\text { Third } \\
\text { Moment }\end{array}$ & $\begin{array}{c}\text { Fourth } \\
\text { Moment }\end{array}$ & $\begin{array}{c}\text { Inner } \\
\text { Product }\end{array}$ & Count Rate & \begin{tabular}{|c|} 
Final \\
Comparator
\end{tabular} \\
\hline pus1112a.mce & 1.09 & 1.28 & 1.80 & 2.21 & 1.04 & 20.00 & 7.78 \\
\hline mant0918.mc & 2.92 & 18.10 & 20.00 & 20.00 & 6.07 & 20.00 & 15.30 \\
\hline mant0919.mc: & 2.04 & 4.27 & 7.49 & 7.83 & 20.00 & 20.00 & 11.71 \\
\hline mant0920.mc & 2.04 & 4.26 & 7.46 & 7.78 & 20.00 & 20.00 & 11.70 \\
\hline eucal1.mca & 2.41 & 8.94 & 20.00 & 20.00 & 7.27 & 20.00 & 14.07 \\
\hline co71108b.asc & 1.71 & 2.69 & 3.00 & 1.73 & 13.47 & 20.00 & 9.35 \\
\hline co601108.asc & 1.71 & 2.70 & 3.08 & 2.02 & 14.73 & 20.00 & 9.65 \\
\hline u23519a.asc & 1.75 & 3.95 & 11.15 & 20.00 & 5.53 & 20.00 & 11.94 \\
\hline u23545a.asc & 1.75 & 3.72 & 9.30 & 20.00 & 6.03 & 20.00 & 11.72 \\
\hline u23545b.asc & 1.75 & 3.72 & 9.35 & 20.00 & 6.08 & 20.00 & 11.73 \\
\hline u23587a.asc & 1.74 & 3.81 & 10.16 & 20.00 & 5.74 & 20.00 & 11.81 \\
\hline man1 107a.asc & 2.06 & 8.48 & 20.00 & 20.00 & 5.55 & 20.00 & 13.91 \\
\hline man1111a.asc & 1.98 & 7.83 & 20.00 & 20.00 & 4.53 & 20.00 & 13.80 \\
\hline u235295b.asc & 1.73 & 3.77 & 10.00 & 20.00 & 5.71 & 20.00 & 11.79 \\
\hline u23529C.asc & 1.82 & 4.10 & 10.97 & 20.00 & 6.21 & 20.00 & 11.96 \\
\hline u23529D.asc & 1.73 & 3.79 & 10.14 & 20.00 & 5.62 & 20.00 & 11.80 \\
\hline pus1112b.mx) & 1.09 & 1.29 & 1.93 & 3.39 & 1.02 & 20.00 & 7.83 \\
\hline pu1113ax.mc & 1.09 & 1.30 & 1.93 & 3.33 & 1.03 & 20.00 & 7.83 \\
\hline pu1113bx.mc & 1.10 & 1.31 & 1.95 & 3.37 & 1.04 & 20.00 & 7.83 \\
\hline vault01x.mca & 2.03 & 5.02 & 12.18 & 20.00 & 3.86 & 20.00 & 12.06 \\
\hline vault02x.mca & 1.02 & 1.13 & 1.45 & 2.05 & 1.02 & 4.66 & 1.57 \\
\hline vault03x.mca & 1.80 & 3.79 & 7.74 & 11.70 & 4.39 & 20.00 & 9.49 \\
\hline vault04x.mca & 1.30 & 2.17 & 4.09 & 6.09 & 1.13 & 20.00 & 8.14 \\
\hline vault05x.mca & 1.46 & 2.72 & 5.86 & 11.24 & 1.30 & 20.00 & 9.06 \\
\hline vault06x.mca & 1.02 & 1.11 & 1.32 & 1.54 & 1.00 & 1.29 & 0.28 \\
\hline vault07x.mca & 1.80 & 5.24 & 20.00 & 20.00 & 1.54 & 20.00 & 13.55 \\
\hline vault08x.mca & 1.53 & 3.05 & 6.91 & 13.36 & 1.38 & 20.00 & 9.60 \\
\hline vault09x.mca & 1.50 & 3.01 & 6.74 & 12.32 & 1.56 & 20.00 & 9.37 \\
\hline vault10x.mca & 2.02 & 4.96 & 11.59 & 18.70 & 4.06 & 20.00 & 11.64 \\
\hline
\end{tabular}

In this report we developed the techniques necessary to make such spectral comparisons and described their application.

If a spectrum is treated as a random variable with a characteristic distribution and/or as an n-dimensional vector with an associated magnitude and direction, then a set of "comparators" can be calculated which are theoretically unique to each spectrum. These comparators can then be used to characterize the radiation source and determine whether or not the source material has been altered.

Using these ideas, we reported on an inventory confirmation "proof of principle" exercise where we went to a real nuclear-material repository and used a small, and very portable, room-temperature CdTe detector to carry out a mock confirmation protocol. The results suggest that this system can become the basis for an instrument that can noninvasively confirm or deny changes in special nuclear material inventories.

\section{Acknowledgements}

The authors wish to thank Joel swanson for his encouragement of this work and to the DOE SO13 sponsors for funding. 
Figure 3A: Spectrum from a camping lantern mantle. The martel fabric is coated with naturally occuring ${ }^{232}$ Th (half-life $=1.4 \times 10 \quad \mathrm{yr}$ ). Under heat, the metal oxide has high emissivity in the visual spectrum and is an ideal light source.

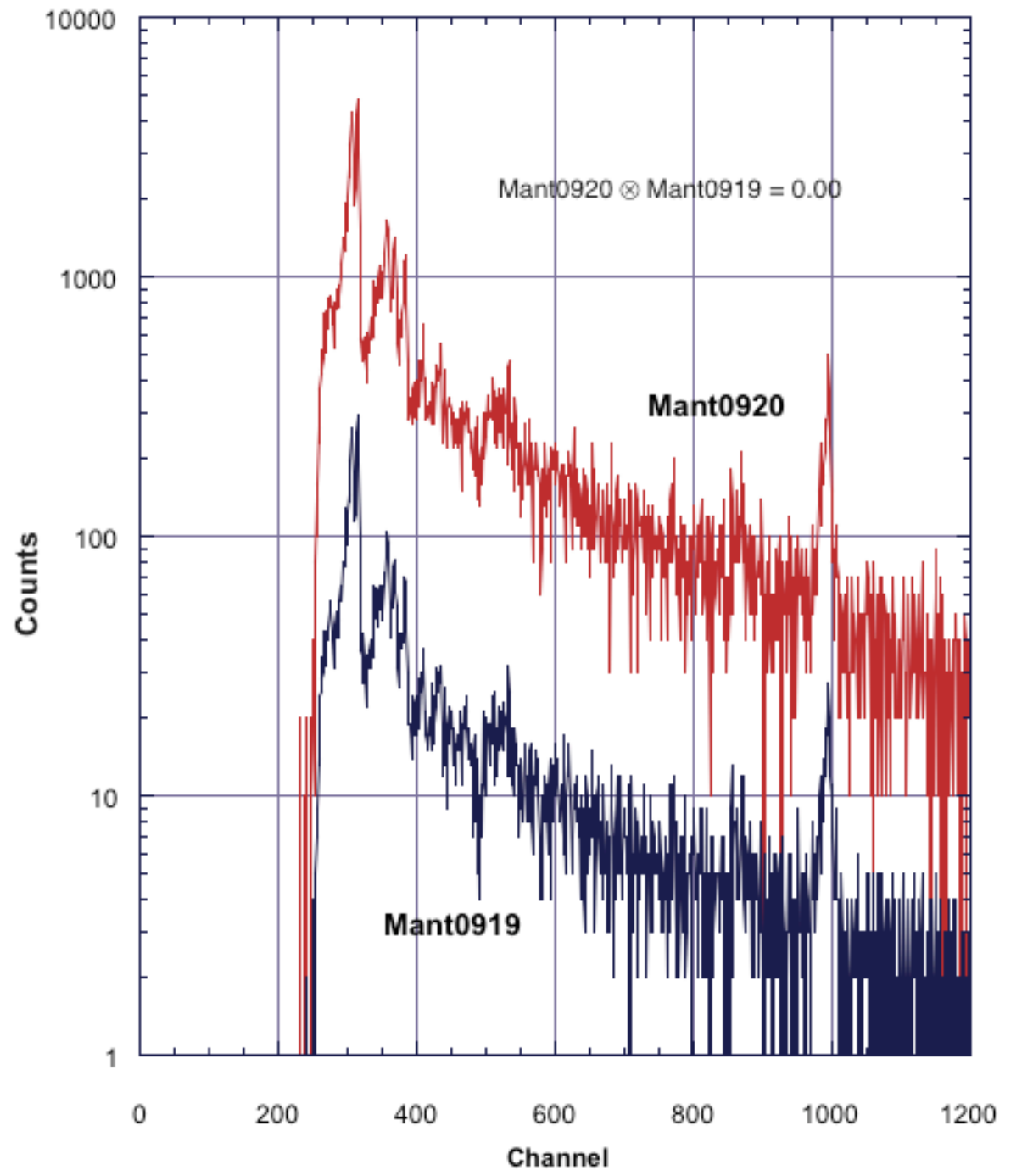


Figure 3B: Spectra U23545a and U23545b are taken from the same container at different times and have a comparator value $=0.01$. Spedrum U23529C represents a different container and has a comparator value $=0.27$. The two different containers differ in their ${ }^{235} \mathrm{U}$ enrichment by $\sim 2 \%$.

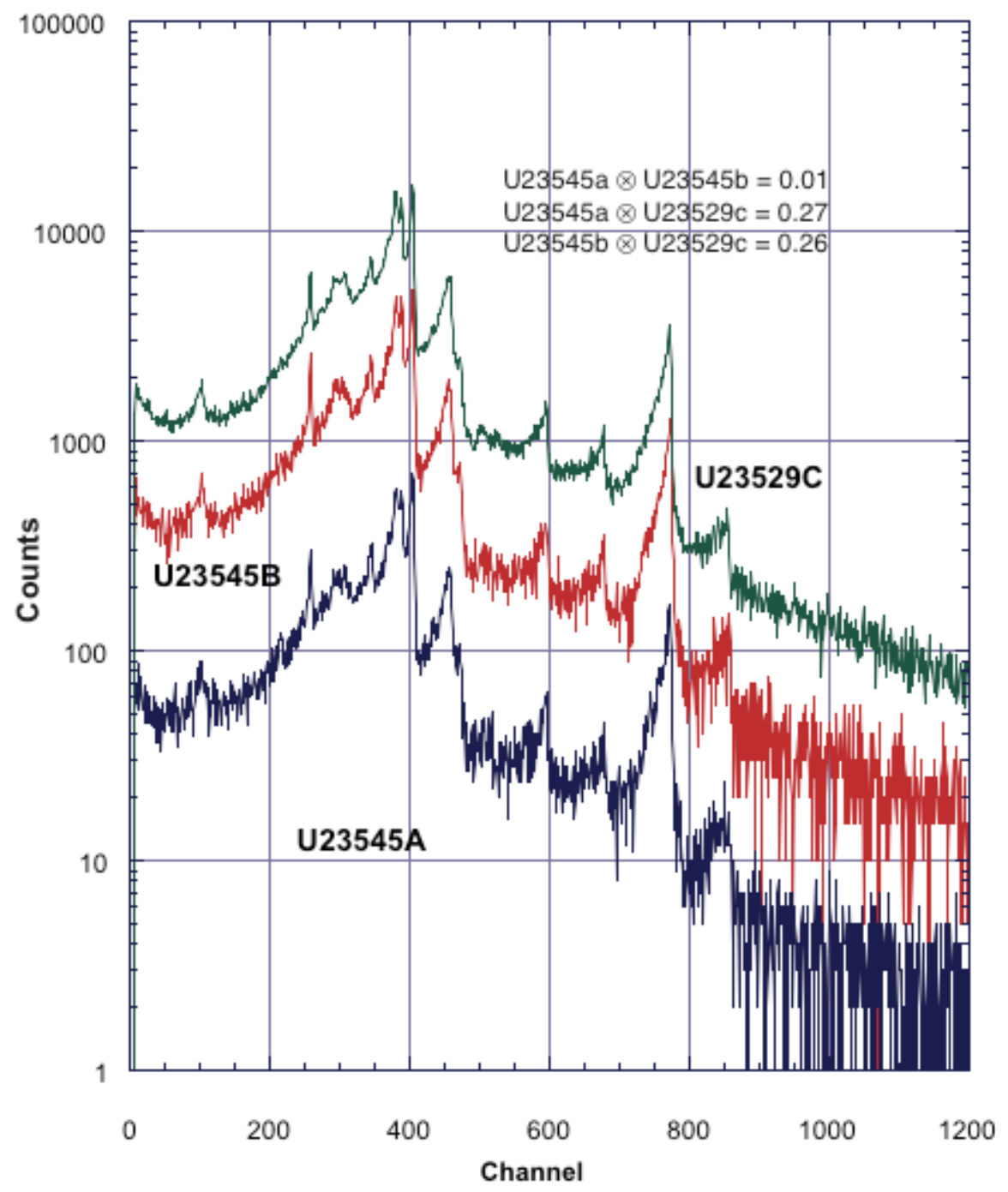


Figure 3C: SNM Vault Comparisons. Spectra Vault01X and Vautl 0X are taken from the same container at different times and have a comparator value $=0.07$. Spectrum Vault04X represents a different container with totally different material and has a comparator value $=2.36$.

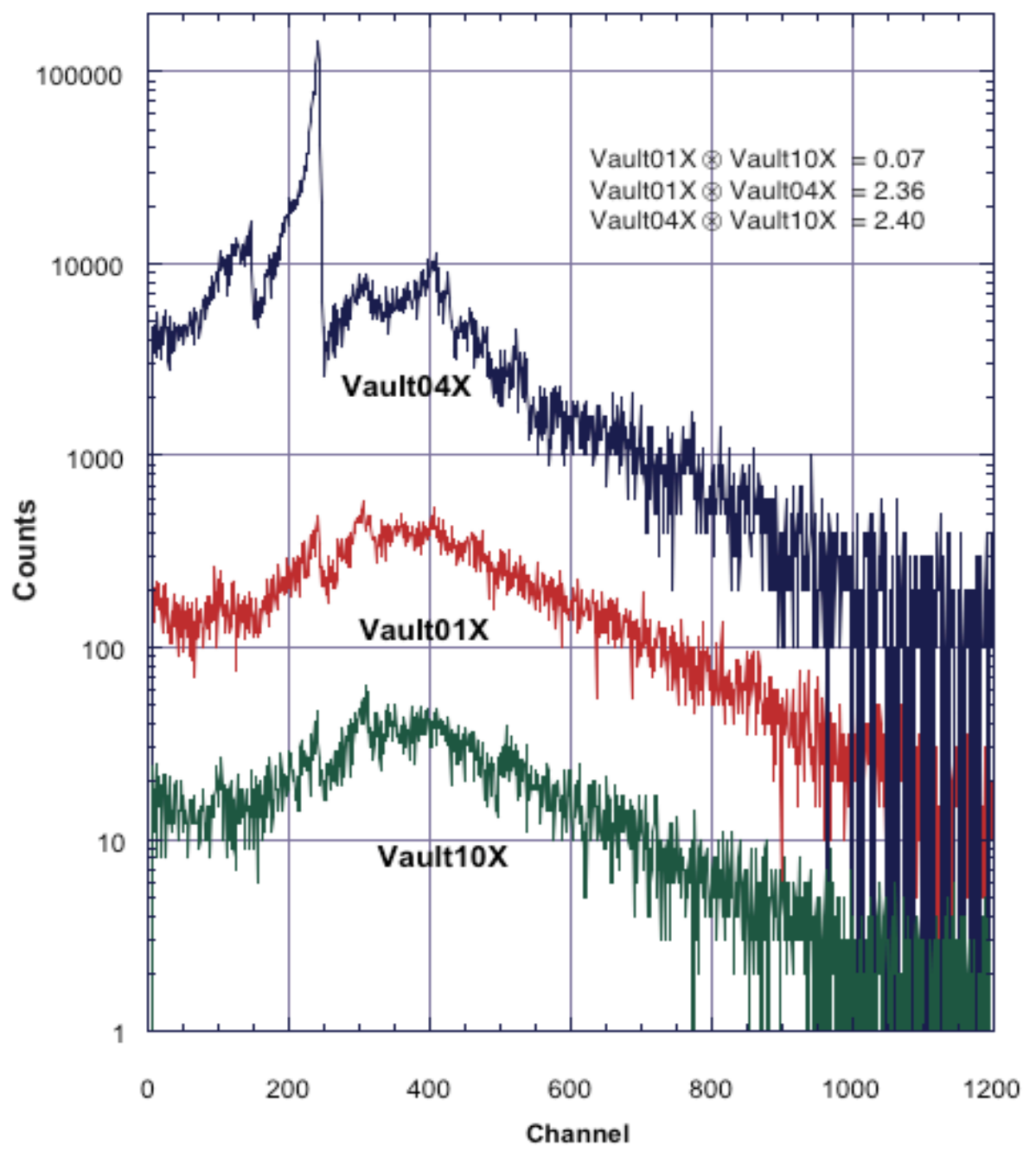




\section{Appendix A}

We previously pointed out that "the detector must be identically positioned relative to the container for subsequent confirmatory measurements." In fact, it is likely that the technique is quite robust relative to small errors in probe positioning relative to the point where the original fiducial measurement is done. This can be seen most clearly by reference to Eqn. (4). The normalization which transforms the spectrum into a probability distribution also approximately normalizes the spectrum's $1 / \mathrm{R}^{2}$ dependence. For an ideal point source - viewed by a point detector - , this is rigorously true because count rate variations would only be caused by $1 / \mathrm{R}^{2}$ dependence.

The idealized situation described above can be shown in the following way. First, if $f\left(x_{i}\right)$ represents the counts recorded in channel $x_{i}$ in time $t$, this is because the radioactive source has actually emitted a total of $\square\left(x_{i}\right)$ quanta that could have been registered in channel $x_{i}$. These two quantities are related through

$$
\xi_{i}^{\&}=\frac{\square_{i}}{t}=R^{2} \cdot \frac{f\left(x_{i}\right)}{t}=R^{2} \cdot \&\left(x_{i}\right)
$$

where $\mathrm{R}$ is the source detector distance and $\$$ is the absolute count rate of the source . Consider now the same ideal source but counted at a new distance $\mathrm{S}$ and for a time $t^{\prime}$, where $t^{\prime} \neq t$ and $S \neq R$, then

$$
\Psi_{i}^{\&}=\frac{\square_{i}}{t \square}=S^{2} \cdot \frac{g\left(x_{i}\right)}{t \square}=S^{2} \cdot \&\left(x_{i}\right)
$$

and where we have assumed that the absolute disintegration rate of the source, $\Psi_{i}^{\&}$, has not changed over the course of the measurement. Rewriting Eqn. (4) and showing the normalization explicitly, we have after substituting Eqn. (9):

$$
Y\left(x_{i}\right)=\& / A=\&\left(x_{i}\right) / \square_{i} \&\left(x_{i}\right)=\frac{\xi_{i}^{\&}}{R^{2}} / \square_{i} \frac{\xi_{i}^{\xi_{i}}}{R^{2}}=\square_{i}^{\xi_{i}} / \square_{i}^{\xi_{i}}
$$

One notes that if we began with Eqn. (10), the same result would be obtained, which proves the relative independence of detector positioning under idealized conditions. In a real case, cancellation of the $1 / \mathrm{R}^{2}$ effects are energy dependent and can vary strongly with source size and details of the packing. The extent to which these effects cancel would need to be evaluated on a case by case basis.

For the present report we simulated a thin, disc source packed into a stainless steel cylindrical container with the following dimensions: Diameter $=18$ ", height $=30$ " and container walls $3 / 32$ " thick. The disk was assumed to be 12 " in diameter; it was placed 
at the center of the container with the areal face perpendicular to the major cylindrical axis and was assumed to emit a gamma-ray spectrum represented by Figure $3 \mathrm{~A}$. We calculated two simulated responses: The first with the detector in contact with the container lid (source-detector separation $a=15$ ") and a second with the probe 5" above the lid (source-detector separation $a=20$ "). The detector response was calculated through

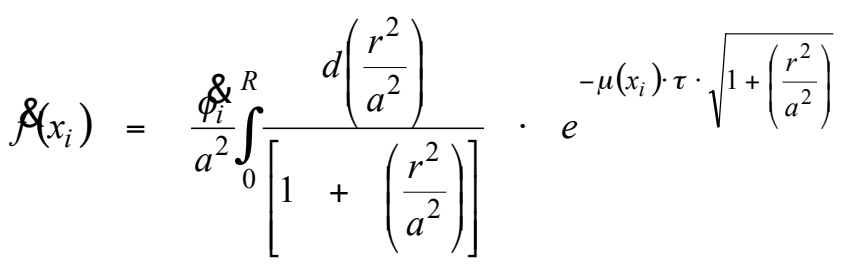

where $R$ is the radius of the disc source, $a$ is the source detector separation, $\square\left(x_{i}\right)$ is the energy dependent mass attenuation coefficient for stainless steel and $\square$ is the thickness of the container lid.

From the recalculated detector responses, we compared the $1^{\text {st }}, 2^{\text {nd }}$ and $3^{\text {rd }}$ moments as given Eqn. (5) and found 1\% variation. Recalling that the source-detector separation is changed by almost $35 \%$, this represents a remarkable insensitivity to that variable. Unfortunately, this same insensitivity cannot be attributed to all the descriptors identified in this report. The count-rate descriptor in Eqn. (8) will not be insensitive to the source-detector separation. This is because the spectral quantities are directly compared and remain unnormalized. In this case, because distance enters the equations as a squared quantity, a $1 \%$ error in detector placement causes at least a $2 \%$ error in comparing count rates.

In general, the exercise investigating the sensitivity of the confirmation protocol to source-detector separation suggests that the technique should still be used with some care. We have, for example, not established sensitivity to source size and geometry, i.e source shape. However, we expect these to be second-order effects. It is interesting that for the five comparators considered here, only one appears overly sensitive to the source-detector separation. 